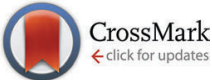

Cite this: DOI: $10.1039 / \mathrm{c} 4 \mathrm{cp} 05486 \mathrm{e}$

\title{
Temperature-cycle microscopy reveals single-molecule conformational heterogeneity $\dagger$
}

\author{
Haifeng Yuan, $\ddagger^{\mathrm{a}}$ Alexander Gaiduk, ${ }^{\mathrm{a}}$ Joanna R. Siekierzycka, ${ }^{\mathrm{a}}$ Satoru Fujiyoshi, \\ Michio Matsushita, ${ }^{b}$ Daniel Nettels, ${ }^{c}$ Benjamin Schuler, ${ }^{c}$ Claus A. M. Seidel ${ }^{d}$ and \\ Michel Orrit*a
}

Our previous temperature-cycle study reported FRET transitions between different states on FRET-labeled polyprolines [Yuan et al., PCCP, 2011, 13, 1762]. The conformational origin of such transitions, however, was left open. In this work, we apply temperature-cycle microscopy of single FRET-labeled polyproline and dsDNA molecules and compare their responses to resolve the conformational origin of different FRET states. We observe different steady-state FRET distributions and different temperature-cycle responses in the two samples. Our temperature-cycle results on single molecules resemble the results in steady-state measurements but reveal a dark state which could not be observed otherwise. By comparing the timescales and probabilities of different FRET states in temperature-cycle traces, we assign the conformational heterogeneity reflected by different FRET states to linker dynamics, dye-chain and dye-dye interactions. The dark state and low-FRET state are likely due to dye-dye interactions at short separations.

Received 25th November 2014 Accepted 27th January 2015

DOI: $10.1039 / \mathrm{c} 4 \mathrm{cp} 05486 \mathrm{e}$

\section{Introduction}

Förster resonance energy transfer (FRET) between FRET labels, which consist of one donor and one acceptor, proceeds via a dipole-dipole interaction. Thus, it is sensitive to distances of 2-10 $\mathrm{nm}$ between FRET labels and it is often referred to as a "spectroscopic ruler". ${ }^{1-3}$ Changes in FRET efficiency reflect changes in conformation or in interactions. ${ }^{4-6}$ By suppressing ensemble averaging, single-molecule FRET has been broadly applied for probing molecular conformational changes and interactions in the past two decades. ${ }^{7-15}$ The FRET efficiency $(E)$, which describes the fraction of energy transferred from the donor to the acceptor, can be calculated ${ }^{2}$ from the intrinsic donor fluorescence lifetime $\left(\tau_{\mathrm{D} 0}\right)$ and its lifetime $\left(\tau_{\mathrm{DA}}\right)$ in the presence of the acceptor:

$$
E=1-\tau_{\mathrm{DA}} / \tau_{\mathrm{D} 0}
$$

Alternatively, the FRET efficiency can also be calculated from the number of photons detected at the donor $\left(n_{\mathrm{D}}\right)$ and acceptor $\left(n_{\mathrm{A}}\right)$ wavelengths: ${ }^{7}$

$$
E=n_{\mathrm{A}} /\left(\gamma n_{\mathrm{D}}+n_{\mathrm{A}}\right)
$$

\footnotetext{
${ }^{a}$ Huygens-KamerlingOnnes Laboratory, Leiden University, Leiden, The Netherlands. E-mail: orrit@physics.leidenuniv.nl

${ }^{b}$ Department of Physics, Tokyo Institute of Technology, Tokyo, Japan

${ }^{c}$ Department of Biochemistry, University of Zurich, Zurich, Switzerland

${ }^{d}$ Institute for Physical Chemistry, Heinrich-Heine-Universität Düsseldorf, Düsseldorf, Germany

$\dagger$ Electronic supplementary information (ESI) available. See DOI: 10.1039/c4cp05486e

\# Present address: Department of Chemistry, KU Leuven, Leuven, Belgium.
}

taking the different fluorescence quantum yields and spectral detection efficiencies via the correction factor $\gamma$ into account. ${ }^{16}$ In either way, it requires at least some hundreds of photons to determine the FRET efficiency. Hence, the time resolution of single-molecule FRET is dictated by photon statistics. Due to the limited emission rate of individual fluorophores and the limited instrumental detection efficiency, the time resolution is limited to a few milliseconds. ${ }^{17}$ Many biological processes, however, take place on a time scale faster than milliseconds. ${ }^{18,19}$ To access fast dynamics of single molecules in real time, one has to increase the emission rate of fluorophores. By directly applying very intense laser excitation ${ }^{20}$ or adding photoprotective agents, ${ }^{21}$ sub-millisecond temporal resolution on single-molecule FRET has been demonstrated. Nevertheless, due to limited dwell time of the molecule in the observation volume, observing the same individual molecules for a long time without immobilizing them onto surfaces is still difficult.

To follow microsecond dynamics on the same molecule for long times without the complication of immobilization, our group proposed a temperature-cycle technique. ${ }^{22}$ This temperature-cycle method is closely related to the well established temperaturejump method at room temperature..$^{23-25}$ In temperature-cycle measurements, the extreme temperatures are chosen such that the dynamics of interest will be frozen at low temperature and be activated at high temperature. A dynamical process can thus be studied as a consecutive series of snapshots of frozen states with controllable time steps. In this method, the time resolution is limited only by the heating and cooling times, which are typically around 3-4 microseconds for the diffraction-limited focal spot 


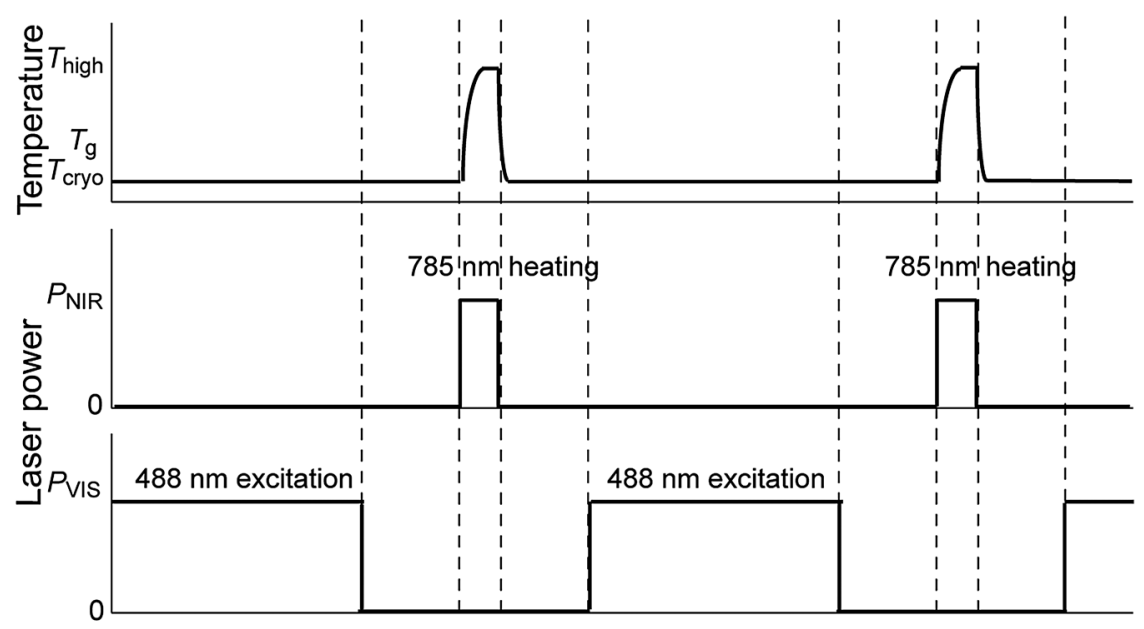

Time

Fig. 1 Scheme of the temperature-cycle method. The evolution of dynamics is allowed or frozen by fast temperature modulations caused by fast intensity modulation of a focused NIR laser beam $(785 \mathrm{~nm}$ ). While the molecule is frozen at the low temperature of the cycle, another laser (at $488 \mathrm{~nm}$ ) excites fluorescence to probe the states of the molecule optically. By alternatively applying sequences of laser illumination periods, the dynamics of the molecule can be recorded as a series of snapshots.

of a microscope. ${ }^{22} \mathrm{~A}$ schematic view of the temperature-cycle method is shown in Fig. 1. In previous work, we demonstrated temperature-cycle microscopy on FRET labeled all-trans polyprolines in glycerol. ${ }^{26}$ All-trans polyproline possesses a helix structure and is rather rigid. It was used as a model system to demonstrate FRET as a spectroscopic ruler. ${ }^{1}$ Embedded in supercooled glycerol, FRET-labeled short polyproline constructs showed broad distributions in FRET values. Furthermore, we observed reversible transitions between a high FRET value and a low FRET value on individual FRET constructs by applying temperature cycles between $170 \mathrm{~K}$ and $250 \mathrm{~K} .{ }^{26}$ Such observations indicate conformational changes of the molecule. However, it proved difficult to determine the origin of the FRET transitions.

Besides the dynamics of the polyproline chain itself, ${ }^{3,27-29}$ many other factors may influence the FRET distribution or time trace, such as dye orientations, dynamics of linkers ${ }^{28,30-35}$ and dye photophysics. ${ }^{36-41}$ Among the above mentioned factors, linker dynamics has received much attention since it can significantly influence FRET experiments. For instance, long and flexible linkers may bring FRET dye pairs close to each other and allow dye-dye interaction to take place. ${ }^{30,37}$ Di Fiori et al. found that dye-dye interactions at short separations lead to multiple-step fluorescence time traces in single-molecule FRET measurements. ${ }^{37}$ However, such interactions also depend on the dye pairs used in experiments. ${ }^{37}$ Recent molecular dynamics simulations ${ }^{28,31}$ have indicated that dynamics of the dye and the linker may induce extra conformational heterogeneity and thus influence measured FRET. The dye molecules with their long and flexible linkers can get into contact with the polyproline chains and form transient interactions. ${ }^{28,31}$

With the hope of unraveling the different factors which influence FRET, we adapted our temperature-cycle microscope to access dye orientations and fluorescence lifetimes by adding a polarization-dependent detection and a time-correlated singlephoton counting (TCSPC) system. To access photon information with additional dimensions, better photon statistics are required. In temperature-cycle measurements, one can simply extend the measurement duration in each low-temperature period to collect as many photons as needed. However, this will reduce the maximum number of temperature-cycle measurements on single molecules before photo-bleaching. In addition, we improved the collection efficiency of the optical system in our cryostat by introducing a hemispherical solid immersion lens (SIL). At room temperature, the numerical aperture (NA) of the optical system can be increased by liquid-immersion techniques. However, liquid immersion can hardly be applied in a low-temperature microscope due to freezing of immersion liquids. Instead, we can use a small hemispherical solid immersion lens (SIL) to ensure optical contact with the glycerol thin film, while keeping the distance between the SIL and the objective adjustable. When the incident light is focused through the hemispherical solid immersion lens $\left(n_{\mathrm{SIL}}\right)$ at its center, the light wavefront will not be distorted by the air-solid interface and will remain spherical. In this way, the NA can be increased by a factor of $n_{\mathrm{SIL}} \cdot{ }^{42-47}$ Moreover, a hemispherical SIL is perfectly achromatic for its center and only slightly chromatic for points in the center's vicinity. Hemispherical SIL's have been used to improve the NA of optical systems in microscopy and spectroscopy applications..$^{43,48,49}$ In addition to a SIL, we use a singlecomponent reflecting objective as a collimating optics for the following reasons. (i) Built from a single piece of fused silica, the single-component reflecting objective possesses superb stability at low temperatures. ${ }^{50,51}$ (ii) It is achromatic since it works with purely reflecting optics. (iii) It has a long working distance of more than $3 \mathrm{~mm}$, thus we can use SILs with diameters of up to $3 \mathrm{~mm}$. Although it has a small numerical aperture (NA, about 0.6 ), the total NA after taking into account the SIL with $n_{\mathrm{SIL}}=2$ can reach 1.2.

Here, we first characterize the hemispherical solid immersion lens and the single-component reflecting objective using 
fluorescent beads. We then apply this optical system for singlemolecule temperature-cycle studies on both FRET-labeled polyproline and double-stranded DNA. By simultaneously measuring the fluorescence intensity and polarization while applying temperature cycles, we compare the results on FRETlabeled polyproline and double-stranded DNA.

\section{Experimental}

The home-built beam-scanning confocal microscope within a cryostat is described in detail in a previous work by Zondervan et $a .^{22}$ A scheme of the optical setup is shown in Fig. 2. A $785 \mathrm{~nm}$ continuous-wave (CW) diode laser (TOPTICA Photonics AG) modulated by an acousto-optic-modulator (AOM, AA opto electronic) is employed as the heating source. The heating beam is directed by a near-infrared (NIR) mirror whose orientation can be adjusted mechanically. An aspheric lens is then employed to focus the heating beam on the chromium film by positioning the lens along the vertical direction with a one-dimension piezo stage (ANPz100, Attocube systems AG). The position of the sample-SIL assembly is controlled three-dimensionally with a stack of close-loop piezo stages (ANPx101, ANPz101, Attocube systems AG). The detailed drawing can be found in a previous work. ${ }^{22}$ The excitation beam from a pulsed $488 \mathrm{~nm}$ laser (BDL-488, Becker\&Hickl GmbH) operating at $50 \mathrm{MHz}$ or at the $\mathrm{CW}$ mode is circularly polarized. After a pair of scanning mirrors and a telecentric system, it enters the cryostat from the bottom window. The excitation beam is then focused by the objective-SIL assembly on the sample-SIL interface. The emitted photons are collected by the same objective and directed to the detection path by a beam splitter. After a $488 \mathrm{~nm}$ notch filter and a $774 \mathrm{~nm}$ shortpass filter, the fluorescence photons are separated by a polarization beam splitter into two paths according to their polarization. Before reaching the avalanche photodiodes (APDs, SPCM-AQR-14, Perkin-Elmer), each of the two polarizations is again divided into two paths according to the wavelength by dichroic mirrors (DM585) and filters (535/50 bandpass filters for donor fluorescence, 630/92 bandpass filters for acceptor fluorescence). In total, the photons are detected in four channels: donor fluorescence of vertical polarization (DV), donor fluorescence of horizontal polarization (DH), acceptor fluorescence of vertical polarization (AV) and acceptor fluorescence of horizontal polarization (AH). The detected fluorescence photons are then recorded by a TCSPC system (TimeHarp200, PicoQuant). A home-built LabView program is used to control the lasers, the AOM and scanning mirrors.

\section{Materials and methods}

\section{Characterization of SIL}

We first use fluorescent beads to characterize the optical system. A suspension of $20 \mathrm{~nm}$ fluorescent beads (FluoSpheres, $0.02 \mu \mathrm{m}$, yellow-green fluorescent 535/575, Invitrogen) from stock was diluted with MilliQ water and mixed with glycerol in a $1: 1$ volume ratio. The glycerol suspension was then spincoated at $6000 \mathrm{rpm}$ for 60 seconds. It yielded a film of about 0.5-2 $\mu \mathrm{m}$ in thickness. A hemispherical SIL (LASF35 glass, $n_{\mathrm{SIL}}=2.02$, Sandoz Fils SA) of $2.5 \mathrm{~mm}$ radius was then contacted to the glycerol film, as shown in Fig. 2. After the sample was inserted into the cryostat, it was dried by alternatingly pumping and flushing with dry helium gas for 30 minutes several times.

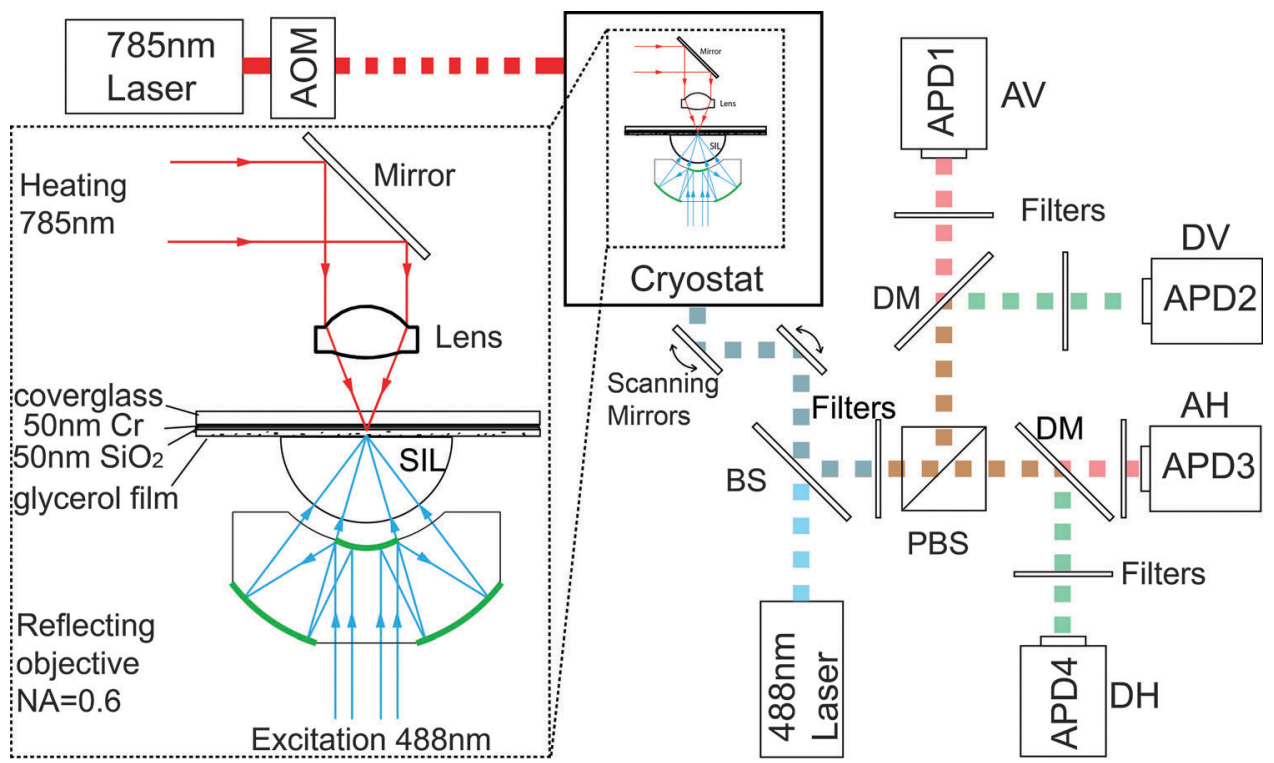

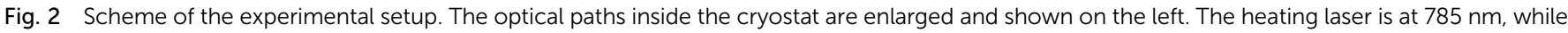

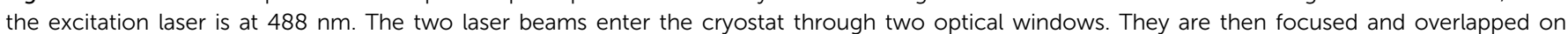

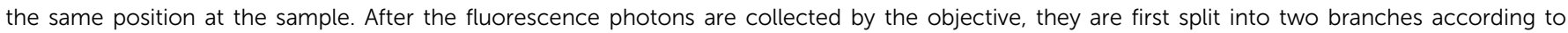

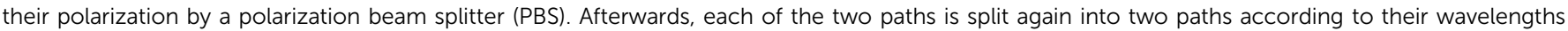

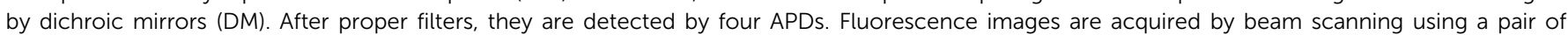
scanning mirrors. 
Afterwards, the sample was kept in a dry and inert helium atmosphere throughout the entire experiment.

Two fluorescence images taken either with the reflecting objective or with the objective-SIL assembly under the same excitation power are shown in Fig. 3(a) and (b). Imaging with the reflecting objective alone, the full-width at half-maximum (FWHM) of fluorescence beads is about $450 \mathrm{~nm}$, as shown in Fig. 3(a). The average fluorescence intensity is 46 photons per $10 \mathrm{~ms}$. While imaging with the objective together with SIL, we observe an average FWHM of about $250 \mathrm{~nm}$ and an average fluorescence intensity of 140 photons per $10 \mathrm{~ms}$. They are compared in Fig. 3(c) and (d). The spatial resolution is improved by a factor of 1.8 , which is very close to the value we would expect $\left(n_{\mathrm{SIL}}=2.02\right)$. The average intensity is improved by a factor of 3 . This value, however, is somewhat lower than the expected value of $4.1\left(n_{\mathrm{SIL}}{ }^{2}\right)$. It can be a consequence of two factors. First, the high-refractive-index SIL is not anti-reflection coated, thus the reflection loss can be significant in both incident and collected light. Taking the reflection loss at the SIL-air interface into account, the expected improvement over collected signals is 3.2 , which is close to that we found in our experiment. Second, the distance between the fluorescent beads and the SIL-glycerol interface is not uniform. Thus, it can introduce variations in the collection efficiencies. At the center of the SIL, we can image at least a $20 \mu \mathrm{m} \times 20 \mu \mathrm{m}$ area without apparent distortions. In experiments hereafter, all our measurements are done within this region.

\section{Single-molecule experiments}

Glycerol was purchased from Sigma-Aldrich and used as received without further purification. Polyprolines with 6 residues (Pro6) or 20 residues (Pro20) were labeled at the ends, ${ }^{3}$ as shown in Fig. 4(a). Alexa488, the donor molecule, was attached to a cysteine residue at the C-terminus of the peptide chain using maleimide chemistry. The acceptor, Alexa594, was attached to a glycine residue at the $\mathrm{N}$-terminus of the peptide chain using a succinimidyl ester. Both the terminal amino acids of the peptide and the dye linkers are highly flexible. We estimate the end-toend contour length of the 6-residue polyproline chain to be about $1.8 \mathrm{~nm}$. Double-stranded DNA (dsDNA) molecules with 48 basepairs were labeled with Alexa488 and Atto647N on different strands, as shown in Fig. 4(b). The sequence of one chain is 5'-d(GGA CTA GTC TAG GCG AAC GTT TAA GGC GAT CTC TGT(Alexa488) TTA CAA CTC CGA)-3'. The sequence of the other chain is $5^{\prime}$-d(TCG GAG TTG TAA ACA GAG AT(Atto647N)C GCC TTA AAC GTT CGC CTA GAC TAG TCC)-3'. The bold letters show
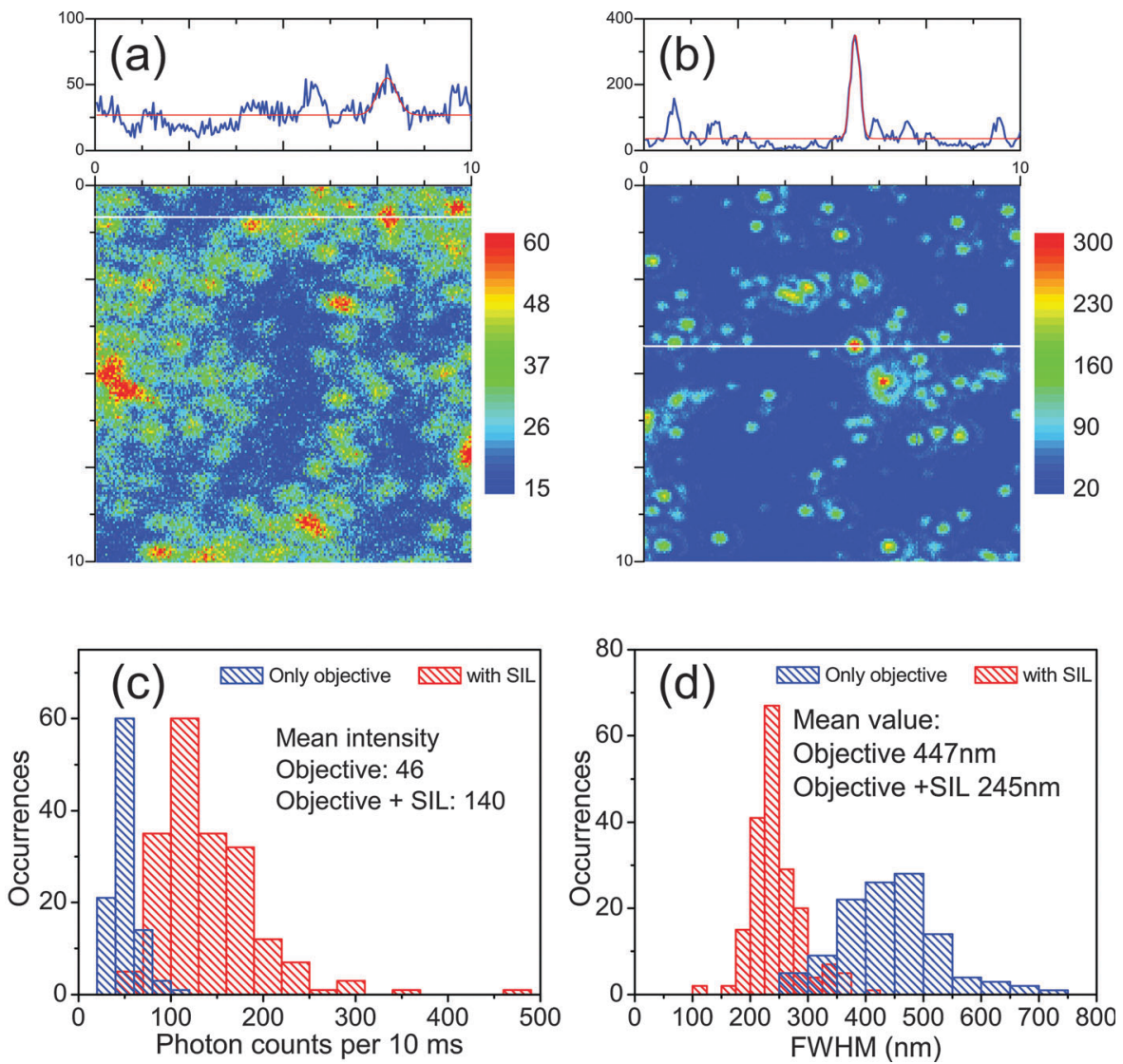

Fig. 3 (a) A fluorescence image of a $10 \mu \mathrm{m} \times 10 \mu \mathrm{m}$ area with fluorescent beads, taken with the reflecting objective. (b) A fluorescence image of a $10 \mu \mathrm{m} \times 10 \mu \mathrm{m}$ area taken with the reflecting objective in combination with the solid immersion lens (SIL). (c) Histograms of bead fluorescence intensities measured with (red) and without (blue) the SIL. The addition of the SIL improves the average intensity from 46 photons per 10 ms to 140 photons per $10 \mathrm{~ms}$. (d) Histograms of FWHMs of fluorescence spots measured with (red) and without (blue) the SIL. The SIL improves the spatial resolution by a factor of almost two, from $447 \mathrm{~nm}$ to $245 \mathrm{~nm}$. 
(a)

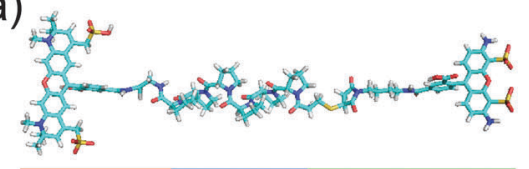

Alexa594 - Gly - [Pro]6 - Cys - Alexa488

(b)

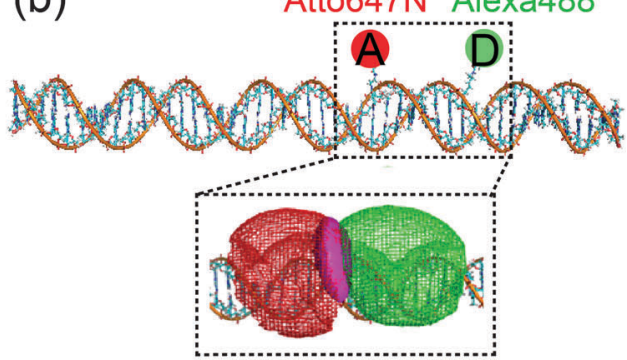

(c)

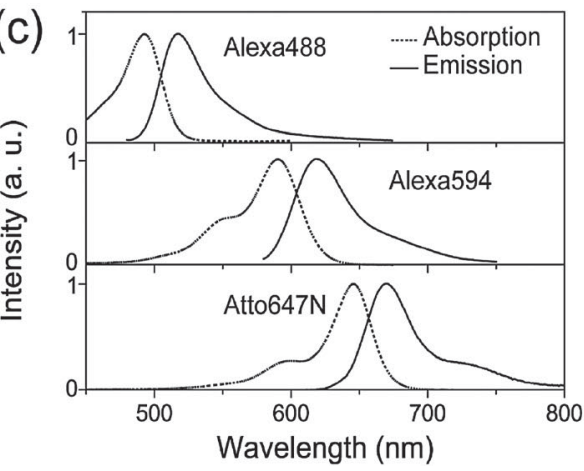

Fig. 4 (a) Molecular structure of the FRET-labeled 6-residue polyproline construct. In the Pro20 construct, the 6-residue polyproline chain is replaced with a 20 -residue polyproline chain. Both the terminal amino acids of the peptide and the dye linkers are highly flexible. (b) Molecular structure of dsDNA sample. The enlarged inset shows the simulated accessible volumes of the donor dye (red) and the acceptor dye (green) of a FRET labeled dsDNA construct. The two dyes were labeled at a separation of 7 basepairs. The magenta points represent the volume in which the dye-dye distance is less than $0.5 \mathrm{~nm}$. (c) Alexa488, Alexa594, Atto647N absorption (dashed) and emission (solid) spectra in water. ${ }^{52}$

the positions where the fluorophores are attached. The labeling positions of the two fluorophores were separated by 7 basepairs, which corresponds to a distance of $2.5 \mathrm{~nm}$ (Fig. 4b). The detailed labeling process was the same as that described in the literature. ${ }^{30}$ Briefly, the internal post-labeling of DNA by fluorescent dyes was accomplished by NHS-esters of dyes (Alexa488 (donor) and Atto647N (acceptor)), which react with the amino group of the long flexible "standard" C6 (hexamethlyen) "linker" of thymine (5-[(aminohexyl)-3-acrylimido $] 2^{\prime}$-deoxy-uridine).$^{30}$ The overall length of the linkage from the attachment point to the center of the chromophore is, thus, given by the length of the linker and the internal chemical structure of the dye and amounts to up to $20 \AA$ A. Absorption and emission spectra of both FRET pairs are shown in Fig. 4(c).

Polyproline samples labeled with Alexa488 and Alexa594 were diluted to $10^{-12} \mathrm{M}$ in $50 \mathrm{mM}$ phosphate buffer solution, the $\mathrm{pH}$ was adjusted to 7.0. dsDNA labeled with Alexa488 and Atto647N was diluted to $10^{-12} \mathrm{M}$ in TRIS (tris(hydroxymethyl)aminomethane) buffer, which contains $20 \mathrm{mM}$ TRIS, $100 \mathrm{mM} \mathrm{NaCl}$ and $10 \mathrm{mM}$ $\mathrm{MgCl}_{2}$. The $\mathrm{pH}$ was adjusted to 7.5. After diluting to desired concentrations in buffer solutions, the sample solutions were mixed with glycerol at a volume ratio of $1: 1$. The sample solutions were then spin-coated onto glass substrates coated with $50 \mathrm{~nm} \mathrm{Cr}$ and $50 \mathrm{~nm} \mathrm{SiO} \mathrm{N}_{2} .60$ second spin-coating at $6000 \mathrm{rpm}$ resulted in a sample film of about $0.5-2 \mu \mathrm{m}$ in thickness. After spincoating, the SIL was contacted to the glycerol-water film. The sample was then mounted onto a three-dimensional piezostage and was inserted into the cryostat. Before cooling down, the samples inside the cryostat were dried by pumping and flushing with dry helium gas several times for half an hour at room temperature. Although the sample was covered by the SIL, which limited evaporation of water from the glycerol film, we did not observe any obvious artifacts due to the small amount of water possibly left in the sample. The sample was then kept in an inert and dry helium atmosphere throughout the entire experiment. The samples were cooled directly from room temperature $(290 \mathrm{~K})$ to $170 \mathrm{~K}$ at a cooling rate of $5 \mathrm{~K}$ per hour.

In steady-state measurements, the cryostat temperature was fixed at $170 \mathrm{~K}$, which is about $20 \mathrm{~K}$ below the glass transition temperature of glycerol. At this temperature the FRET constructs' motions are frozen, thus they show almost constant FRET efficiency. The FRET efficiency $(E)$ is calculated from fluorescence time traces using the following equation:

$E=\frac{n_{\mathrm{AH}}+n_{\mathrm{AV}}-\left\langle B_{\mathrm{AH}}\right\rangle-\left\langle B_{\mathrm{AV}}\right\rangle}{\gamma\left(n_{\mathrm{DH}}+n_{\mathrm{DV}}-\left\langle B_{\mathrm{DH}}\right\rangle-\left\langle B_{\mathrm{DV}}\right\rangle\right)+n_{\mathrm{AH}}+n_{\mathrm{AV}}-\left\langle B_{\mathrm{AH}}\right\rangle-\left\langle B_{\mathrm{AV}}\right\rangle}$,

where $n_{\mathrm{AH}}, n_{\mathrm{AV}}, n_{\mathrm{DH}}, n_{\mathrm{DV}}$ are the detected fluorescence intensities from the corresponding APDs and $\left\langle B_{\mathrm{AH}}\right\rangle,\left\langle B_{\mathrm{AV}}\right\rangle,\left\langle B_{\mathrm{DH}}\right\rangle$, $\left\langle B_{\mathrm{DV}}\right\rangle$ are their corresponding backgrounds. $\gamma=\frac{\Phi_{\mathrm{A}} \eta_{\mathrm{A}}}{\Phi_{\mathrm{D}} \eta_{\mathrm{D}}}$ is the correction factor for differences in dye quantum yields $\left(\Phi_{\mathrm{A}}, \Phi_{\mathrm{D}}\right)$ and collection efficiencies $\left(\eta_{\mathrm{A}}, \eta_{\mathrm{D}}\right)$. In the current experimental setup, $\gamma=0.6$ for Alexa488 and Alexa594 while $\gamma=0.7$ for Alexa488 and Atto647N. The crosstalk from the donor channel to the acceptor channel is less than $10 \%$. Therefore, we only take the FRET efficiency values above 0.15 into account. The donor fluorescence lifetime is measured by deconvoluting the instrumental response function (IRF) from the TCSPC histogram and mono-exponential fitting using a maximum likelihood estimation method. ${ }^{53}$ IRF is measured by collecting scattered light from a clean glass coverslide. Linear dichroism $(D)$ is calculated using the equation below:

$$
D=\frac{n_{\mathrm{H}}-\left\langle B_{\mathrm{H}}\right\rangle-\left(n_{\mathrm{V}}-\left\langle B_{\mathrm{V}}\right\rangle\right)}{n_{\mathrm{H}}-\left\langle B_{\mathrm{H}}\right\rangle+n_{\mathrm{V}}-\left\langle B_{\mathrm{V}}\right\rangle},
$$

where $n_{\mathrm{H}}$ is the fluorescence intensity at the horizontal polarization, $n_{\mathrm{V}}$ is the fluorescence intensity at the vertical polarization and $\left\langle B_{\mathrm{H}}\right\rangle,\left\langle B_{\mathrm{V}}\right\rangle$ are their corresponding backgrounds.

In temperature-cycle measurements, the same parameters were applied to both Pro6 and dsDNA samples. The cryostat temperature was kept at $170 \mathrm{~K}( \pm 0.1 \mathrm{~K})$ throughout the entire experiment. The heating power was set to reach a higher temperature of $290 \mathrm{~K}$ in a temperature cycle according to our 
previous calibration. ${ }^{22,26}$ In each temperature cycle, the heating beam was applied for $20 \mu$ s and the $50 \mathrm{MHz}$-pulsed excitation beam was applied for $500 \mathrm{~ms}$ or CW excitation was applied for $60 \mathrm{~ms}$.

\section{Results}

The conformations of FRET constructs are frozen in glycerol at $170 \mathrm{~K}$. Therefore, they show constant signals in both donor and acceptor fluorescence. A typical fluorescence time trace of a Pro6 construct is shown in Fig. 5(a). Although noisy, the fluorescence intensities in donor (light blue) and acceptor (red) channels are almost constant until a sudden drop in both intensities. This digital event is the signature that the fluorescence signal stemmed from a single construct. The single-step drop in fluorescence signals is most likely due to photobleaching of the donor fluorophore. Using average intensities at donor and acceptor channels, we estimate the FRET efficiency of this molecule to be 0.63 . The donor fluorescence lifetime decay of the same molecule is shown in Fig. 5(b). Fitting the lifetime decay with a mono-exponential function after deconvolution of the instrumental response function (IRF), we obtain a donor fluorescence lifetime of $2.1 \mathrm{~ns}$.
The correlation between FRET efficiencies and donor fluorescence lifetimes is shown in Fig. 5(c) and (d). In Fig. 5(c), each green spot represents a measurement on a single molecule. The horizontal position corresponds to its donor fluorescence lifetime and the vertical position corresponds to its FRET efficiency. The red line shows the expected correlation between FRET efficiency and donor fluorescence lifetimes, $E=$ $1-\tau_{\mathrm{DA}} / \tau_{\mathrm{D} 0}\left(\tau_{\mathrm{D} 0}=3.5 \mathrm{~ns}\right.$, measured on Alexa488-labeled polyproline). The correlation observed in our measurements on 98 single Pro6 molecules is weak and shows deviations from theory for molecules with high FRET efficiencies. That is probably due to their low donor fluorescence intensities. For this reason, the donor fluorescence lifetimes of molecules with FRET efficiencies above 0.9 are not shown in Fig. 5(c). Similar measurements on Pro20, which has a longer interdye distance, shows less deviations in FRET efficiencies and randomly distributed donor fluorescence lifetimes in Fig. 5(d).

Although the distances between the labeling positions of the donor and acceptor, $1.8 \mathrm{~nm}$ for the Pro6 sample and $2.5 \mathrm{~nm}$ for the dsDNA sample, are very similar, we observed surprisingly different FRET efficiency distributions at steady state $(T=170 \mathrm{~K})$. On the Pro6 sample, a broad FRET efficiency distribution with a significant low-FRET population was found, as shown in Fig. 6(a). This observation is consistent with our previous experiments. ${ }^{26}$
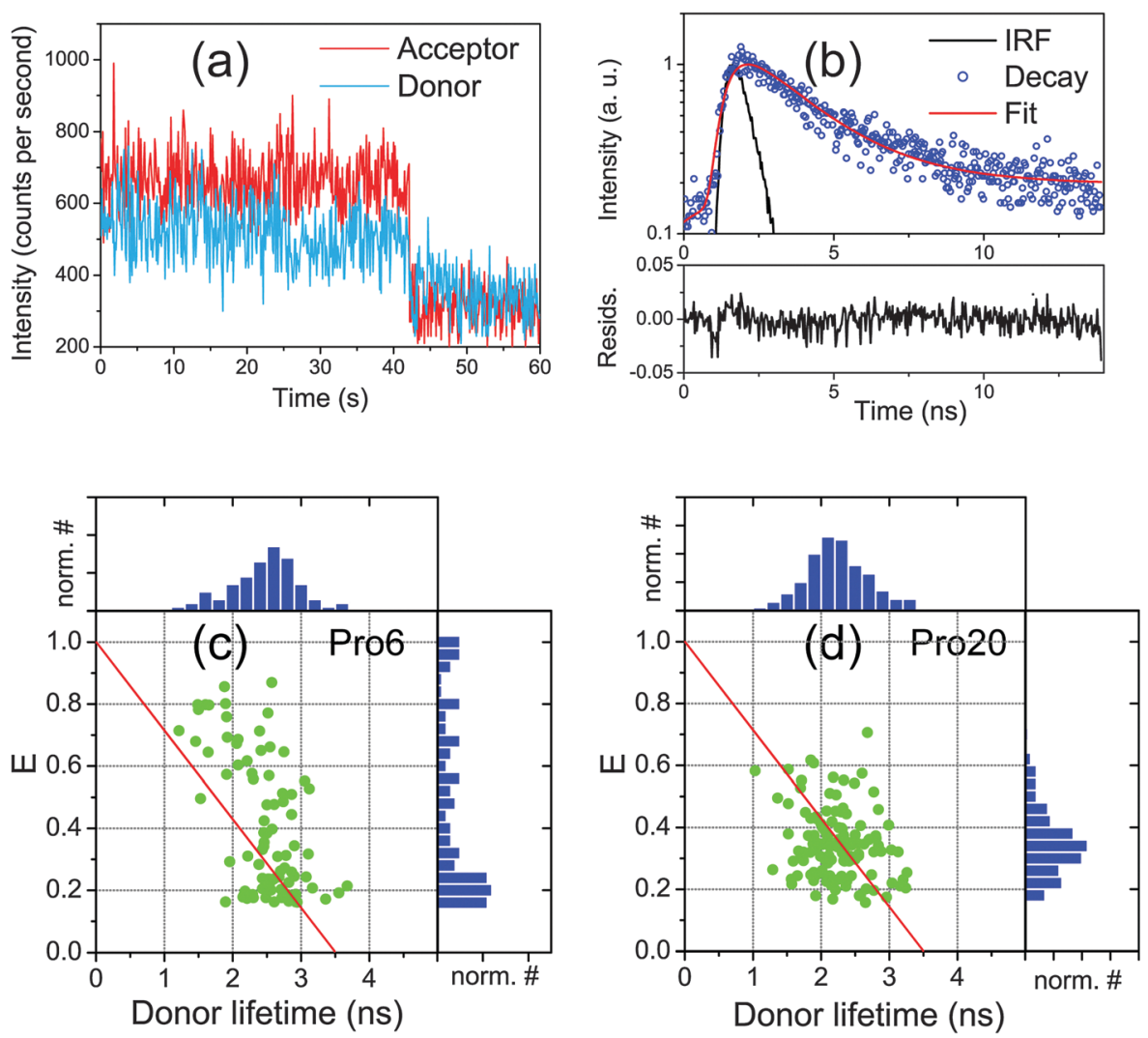

Fig. 5 (a) Typical steady-state fluorescence time traces recorded at $170 \mathrm{~K}$ on a single FRET construct (Pro6) under $50 \mathrm{MHz}$ pulsed excitation. Both the donor (light blue) and the acceptor (red) show almost constant fluorescence intensities before bleaching. The intensities yield a FRET efficiency of 0.63 . (b) The donor fluorescence lifetime decay (blue circles) of the fluorescence trace in (a) and its mono-exponential fit (red) after deconvolution of the instrument response (black). (c) and (d) Correlation between donor fluorescence lifetimes and FRET efficiencies $E$ measured on single Pro6 and Pro20 molecules. The red line represents the expected correlation expressed in eqn (1), where $\tau_{\text {DO }}=3.5 \mathrm{~ns}$. 

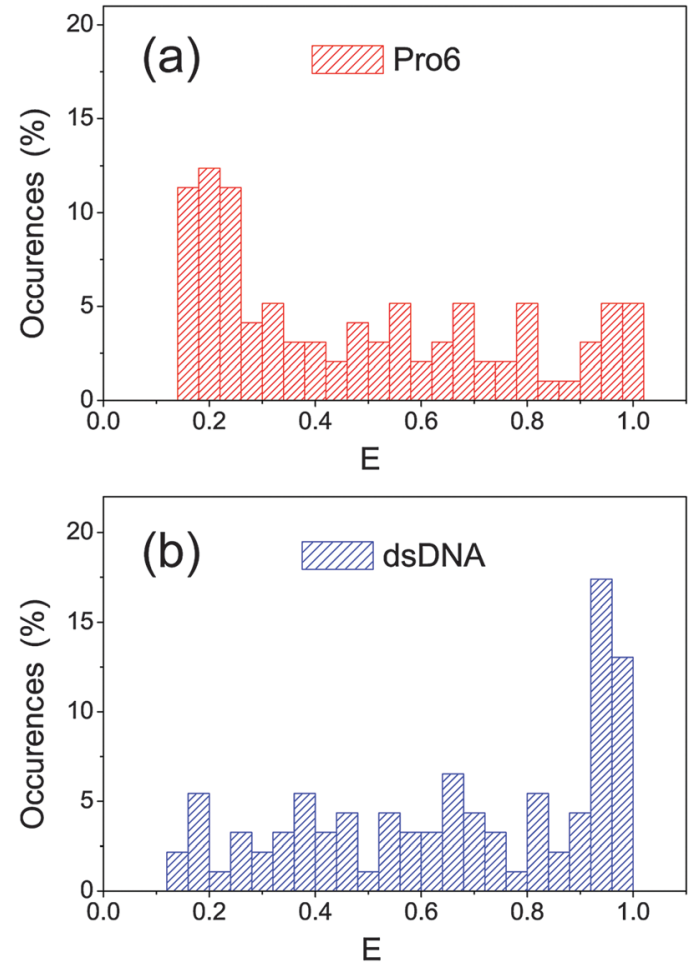

Fig. 6 (a) and (b) Steady-state FRET histograms of Pro6 and dsDNA at $170 \mathrm{~K}$, respectively.

On the contrary, the dsDNA sample shows a large population at almost unity values, as shown in Fig. 6(b). Despite some population at low-FRET values, this distribution agrees qualitatively with Förster theory's prediction for short interdye distances. ${ }^{21}$

Temperature cycles between $170 \mathrm{~K}$ and $290 \mathrm{~K}$ were applied on both Pro6 and dsDNA samples. Three reconstructed temperaturecycle traces are shown in Fig. 7 and 8. In Fig. 7(a), a Pro6 molecule experienced more than 700 temperature cycles before bleaching. It showed several transitions among three different FRET states: a dark state (D), a low-FRET state (L) and a high-FRET state (H). We can recognize these three FRET states from the acceptor-donor scatter plot in Fig. 7(b). In the dark state, both the donor and the acceptor remain dark in fluorescence.

The low-FRET state shows high intensities in the donor fluorescence (light blue) and low intensities in the acceptor fluorescence (red). In contrast, the high-FRET state shows a low donor fluorescence intensity but a high acceptor fluorescence intensity. The observation of three states differs from previous reports of two FRET states only (low-FRET and high-FRET) on Pro6 constructs. This may be due to the different temperaturecycle parameters applied during measurements. ${ }^{26}$ In this work, we applied temperature cycles to higher temperatures $(290 \mathrm{~K}$ instead of $250 \mathrm{~K}$ ) with longer heating durations (20 $\mu$ s instead of $10 \mu \mathrm{s}$ ). This allows the molecule's conformation to evolve for longer times at higher temperature. Thus, the transition to a dark state may not have been revealed in that previous study. Moreover, the dark state will not be reflected in the steady-state FRET histogram. The FRET histogram in Fig. 7(c) shows a broad distribution and a major population in low-FRET values.
Fig. 7(d) shows the correlation of successive FRET values (transition mapping), which indicates that the transitions between different states are relatively slow. Besides FRET efficiencies, the donor fluorescence lifetime is also measured for each temperature cycle. The donor fluorescence lifetime trace in Fig. 7(e) shows some weak changes. However, given the limited number of photons in each measurement (on average less than 100 photons, as shown in Fig. 7(f)), the uncertainty due to shot noise is about 0.32 ns. Therefore, to gain more information from lifetime measurements, photon statistics still needs to be improved, for instance by extending the excitation duration in between each temperature cycle. Nevertheless, the FRET histogram and the lifetime histogram shown in Fig. 7(c) and (e) both resemble the principal features of the steady-state distribution of all Pro6 molecules shown in Fig. 5(c).

Under the same temperature cycles, a dsDNA molecule showed reversible transitions between a low-FRET state and a high-FRET state during 3200 temperature cycles without the presence of any dark state, as shown in Fig. 8(a). Intensities of donor and acceptor fluorescence showed anticorrelated changes in the time traces. The acceptor fluorescence intensity occasionally dropped to the background level while donor fluorescence increased to about 15 photons per $10 \mathrm{~ms}$. After 10 fast switches between the low-FRET and high-FRET states, the acceptor bleached. We also observed a recovery in donor fluorescence right after the acceptor bleached. The donor finally bleached after a few dozens more temperature cycles. The switching between $E=0.2$ and $E=0.8$ is also visible in the FRET trace shown in Fig. 8(c). However, another dsDNA molecule showed a different behavior, as shown in Fig. 8(b). During the 2500 temperature-cycles, this dsDNA molecule showed high intensities in acceptor fluorescence before it bleached. Instead of switching between low-FRET and high-FRET states, it showed several different intensity levels in the acceptor fluorescence time trace. Meanwhile, it showed very low intensities in donor fluorescence without much fluctuation ( $E=0.9$ in Fig. 8(d)). In the beginning 1500 temperature cycles, the acceptor fluorescence was about 23 photons per $10 \mathrm{~ms}$. It then decreased to around 20 photons per $10 \mathrm{~ms}$ and lasted for about 800 temperature-cycles before further decreasing to 10 photons per $10 \mathrm{~ms}$. Shortly, it recovered to 20 photons per $10 \mathrm{~ms}$ and bleached. After the acceptor bleached, there was no recovery in donor fluorescence.

Besides FRET efficiencies, orientations of dyes were monitored by their fluorescence linear dichroism. However, in the temperature-cycle measurements on Pro6 and dsDNA, either the donor or the acceptor showed high fluorescence intensities alternatively. Thus, it is difficult to follow the orientations of both dyes. Nevertheless, we observed reorientation of the strongly emitting dye in dsDNA because its intensity is relatively constant. Fig. 8(e) shows the linear dichroism trace of the acceptor dye in the dsDNA molecule. The noisy trace shows fluctuations that are larger than shot noise in linear dichroism, indicating changes in the acceptor's in-plane orientation. It does not show any obvious correlation to the measured FRET efficiencies. To characterize the rotational dynamics, the autocorrelation of the linear dichroism trace is calculated and is shown in Fig. 8(f). It shows an almost flat correlation curve, 

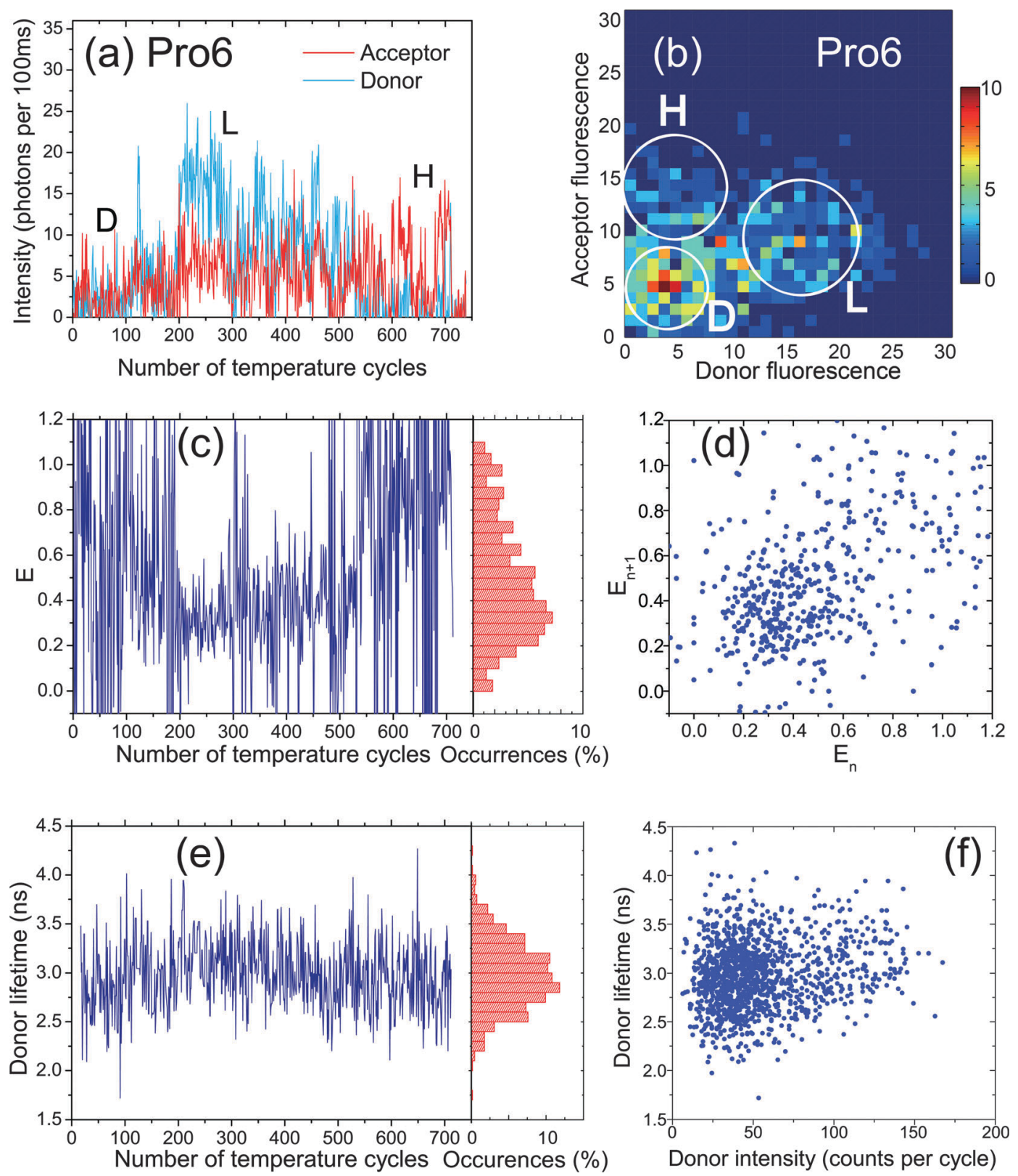

Fig. 7 In each temperature cycle, the heating beam was applied for $20 \mu \mathrm{s}$ and the $50 \mathrm{MHz}$-pulsed excitation beam was applied for 500 ms. (a) A reconstructed temperature-cycle time trace of donor (light blue) and acceptor (red) fluorescence on a single Pro6 molecule. (b) The correlation map of the donor fluorescence intensities against the acceptor fluorescence intensities of the trace shown in (a). The high-FRET state (H), the low-FRET state (L) and the dark state (D) are highlighted in white cycles. (c) The FRET efficiency temperature-cycle trace of the trace in (a). The average uncertainty on $E$ caused by shot noise, and calculated from the number of photons in each measurement, is about 0.14. (d) The jump history of FRET efficiency. (e) The temperature-cycle trace of donor fluorescence lifetime measured in each temperature cycle of the trace in (a). Calculating from the number of photons in each measurement (about 100 photons per decay), ${ }^{53}$ shot noise induces 0.32 ns uncertainty in lifetime measurements. (f) The correlation map of the donor fluorescence intensity against lifetime.

thus indicating that a fast rotational dynamics takes place during each temperature cycle. One possible reason is that the temperature-cycle parameters applied here are not optimal for probing rotational dynamics. The other possible reason is the low signal-to-noise ratio of our fluorescence traces. Both of the above can be further optimized in the future by adjusting the temperature-cycle parameters (extending the excitation time or shortening the heating time in each temperature-cycle).
To further verify that molecules can be brought back to motion by applying heating cycles, gold nanorods' rotational dynamics ${ }^{54}$ was studied using similar temperature cycles (see Fig. S1 and S2, ESI $\dagger$ ). In this demonstration, a gold nanorod's rotational diffusion was followed by its photoluminescence linear dichroism while applying temperature cycles which brought it to room temperature for $0.1 \mathrm{~ms}$ in each cycle. We found that the photoluminescence signals in the two different 

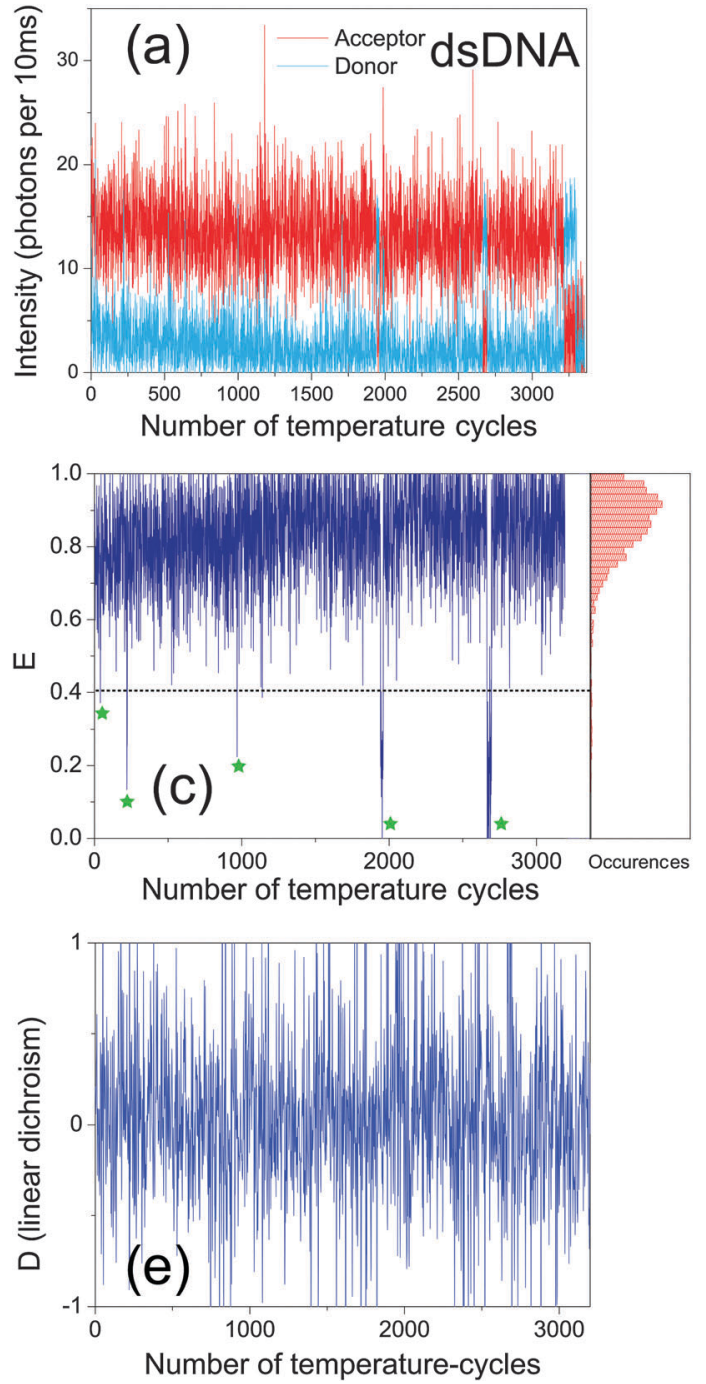
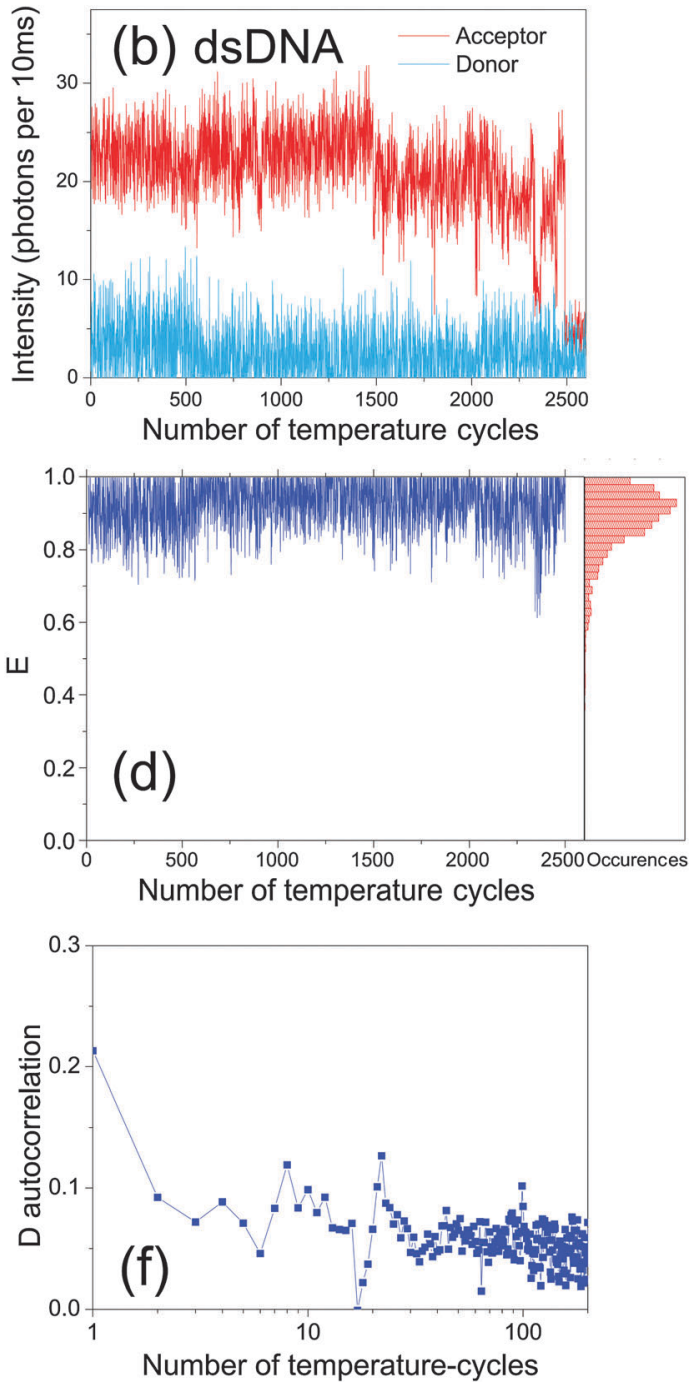

Fig. 8 (a) A reconstructed temperature-cycle time trace of donor (light blue) and acceptor (red) fluorescence on a single dsDNA molecule recorded under CW laser excitation (60 ms after each temperature cycle). (b) Another temperature-cycle time trace on a single dsDNA molecule. (c) Calculated FRET efficiency trace of the fluorescence traces in (a). The average uncertainty induced by shot-noise on $E$ is about 0.10 , calculating from the number of photons in each measurement. The FRET transition events are highlighted with green stars. A threshold of 0.4 , indicated with a dashed line, is applied on the FRET values. (d) Successive values of the efficiency after each thermal cycle and FRET histogram for the traces in (b). (e) Linear dichroism trace of the acceptor in (a). The average shot-noise induced uncertainty on $D$ is about 0.18 , calculating from the number of photons in each measurement. (f) The autocorrelation of the linear dichroism trace in (e), showing no clear correlation time except for shot noise in the first bin.

polarizations were strongly anti-correlated, indicating rotational motions of the gold nanorod upon temperature cycles.

\section{Discussion}

We have shown single-molecule FRET measurements on polyproline and dsDNA molecules both in steady-state and under temperature cycles. In the following, we first discuss different results of steady-state measurements. Embedded in glassy glycerol, the guest molecules' conformations are frozen. Thus, their conformational heterogeneity is preserved and can be reflected by FRET measurements. Broad FRET distributions were found in both Pro6 and dsDNA molecules. However, Pro6 molecules showed a major population in low FRET values while
dsDNA molecules showed mainly high FRET values. Several possible factors may lead to such different results: (i) differences in chain dynamics of polyproline and dsDNA; (ii) different acceptors used for FRET; (iii) differences in volumes accessible to the dyes. Let us discuss them one by one in the following.

First of all, we comment on the differences in chain dynamics. All-trans polyproline is known to possess a helix structure and is rather rigid. ${ }^{1}$ Its persistence length is reported to be longer than $5 \mathrm{~nm} .^{28}$ Although cis-trans transitions can take place between proline residues ${ }^{55,56}$ and lead to a shorter persistence length than that expected for the all-trans conformation, ${ }^{27}$ these transitions are slow. ${ }^{28,57}$ The double-helix chain of dsDNA is even more rigid than polyproline chains. It has a stable structure and its persistence length is $50 \mathrm{~nm}$. Both Pro6 and dsDNA used in this work are shorter than their 
persistence lengths. Hence, we can consider both of them as rigid rods.

We then comment on the different acceptors. Though Pro6 and dsDNA are labeled with different acceptors (Alexa594 and Atto647N), their labeling positions are almost equally separated from that of the donor. In Pro6 molecules, donor and acceptor dyes are labeled at two ends of the peptide chain of 6 residues. The end-to-end contour length of the polyproline chain is about $1.8 \mathrm{~nm}$. Instead of labeling at two ends, dyes are labeled in the middle of the dsDNA chain but with a similar separation of 7 basepairs, which corresponds to about $2.5 \mathrm{~nm}$. Both distances between labeling positions are much shorter than the Förster radii of FRET-pairs (about $5 \mathrm{~nm}$ ). Hence, we would expect the major population to be at high FRET values for both samples.

The last but the most likely reason for the different FRET distributions found on Pro6 and dsDNA is the difference in the accessible volumes for dyes. As mentioned above, dyes are attached at two ends of the polyproline chain but in the middle of the dsDNA chain. Thus, dyes in different samples can access different volumes as already demonstrated in several simulations and experiments. ${ }^{28,30,31}$ Attached at the ends of the peptide chain, the dyes in Pro6 can fully explore the volume around it, within the length of the linker. ${ }^{28,31}$ Limited by both the linkers and double-helix around them, dyes attached on dsDNA have limited access to surroundings ${ }^{30}$ (Fig. $4 \mathrm{~b}$ and Fig. S3, ESI $\dagger)$. The long ( $2 \mathrm{~nm}$ ) and flexible linkers used in dsDNA constructs yield significant uncertainties in positions of dyes and their quenching environment, as indicated in the overlapping accessible volumes of the donor dye and the acceptor dye shown in Fig. 4b and Fig. S3 (ESI $\dagger$ ).

In addition, the dynamics of linkers can be different due to their properties, thus different interactions with the surroundings, which also influence the measured FRET distributions. ${ }^{30}$

When the dyes are labeled at short separations, they could possibly come very close to each other (even into contact) to allow dye-dye interaction to take place. ${ }^{28,31,37,58}$ In the molecular dynamics simulation on polyproline labeled with Alexa488 and Alexa594, Best et al. and Hoefling et al. found that the long and flexible linker can bend in and bring the dye very close to the polyproline chain or even close to the other dye. ${ }^{28,31}$ The presence of such conformations can easily influence the observed FRET distribution of Pro6 by introducing extra interactions (either between dyes and polyproline chain or between dyes) which are not described by Förster theory. In dsDNA, similar dye-dye interactions can also occur, ${ }^{37}$ but less frequently, because of the extra limitations on dye accessible volumes by DNA structures. Furthermore, variations of measured FRET due to such conformational states strongly depend on the interdye distances. Dye-dye interaction will be less likely to occur if the interdye distance is longer. This is evident from the FRET distribution observed on Pro20, which has a longer interdye distance (about $7 \mathrm{~nm}$ ). Pro20 showed a steady-state FRET distribution which is in fairly good agreement with that predicted by Förster theory. A similar dependence of dye-dye interaction on the polyproline lengths was also observed in room-temperature measurements in water solution (Fig. S4, ESI $\dagger$ ).
Our temperature-cycle measurements on Pro6 revealed three fluorescence states including a dark state, a low-FRET state and a high-FRET state. On dsDNA, a low-FRET state and a highFRET state were found by applying temperature cycles. In the steady-state measurements, we can readily recognize the lowFRET state and the high-FRET state from FRET histograms of both samples (Fig. 6). However, the temperature cycle measurements on Pro6 revealed a third state in which both the donor and acceptor are dark in fluorescence. This dark state, otherwise, can never be revealed in ensemble-averaged steady-state measurements. Moreover, we observed multiple-level intensities in the acceptor fluorescence in the high-FRET state of dsDNA. The transitions between different FRET states imply conformational changes.

The snapshots with controlled step sizes in temperature cycle experiments may provide more information on the dynamical processes than real-time measurements. Let us first consider the time step of each temperature cycle. The molecule under investigation was brought to $290 \mathrm{~K}$ by a $20 \mu \mathrm{s}$ heating pulse. Neglecting dynamics during the heating time taken to reach the aimed temperature ${ }^{22}(4 \mu \mathrm{s})$, the molecule evolves for about $16 \mu \mathrm{s}$ at $290 \mathrm{~K}$ before being again frozen. Taking into account glycerol's high viscosity ${ }^{59}$ at this temperature, which is about 1600 times larger than that of water at $290 \mathrm{~K}$, and scaling time with viscosity, this evolution corresponds roughly to about $10 \mathrm{~ns}$ in water at room temperature.

What about the dyes' rotational dynamics during this time? dsDNA samples clearly show complete reorientation of the acceptor from cycle to cycle, as can be seen from the linear dichroism traces in Fig. 8(e). However, rotational times of dyes labeled to dsDNA have been reported to be less than $1 \mathrm{~ns}$ in water solution at room temperature. ${ }^{30}$ Therefore, it is not surprising that temperature cycles equivalent to $10 \mathrm{~ns}$ of rotational diffusion in water cannot resolve such fast rotational dynamics. Therefore, temperature cycles are either too long or at a too high temperature to resolve rotational motion on the dye labels. The temperature-cycle parameters were not optimized for that purpose, which would require further investigation.

The rotational dynamics of donor and acceptor dyes influences the FRET efficiency because FRET depends on the relative orientation of the interacting dipoles as well as on the distance between the dipoles. At short interdye distances of $3 \mathrm{~nm}$ and $4 \mathrm{~nm}$, we expected to observe mainly FRET values between 0.7 and 1.0 for random dye orientations. ${ }^{2,26,60,61}$ Therefore, the probability to observe FRET changes induced by dyes' rotational dynamics will be very low in our Pro6 and dsDNA samples. It also explains why we did not observe any correlation between dye reorientation and measured FRET on dsDNA molecules. Thus, the FRET transitions observed upon temperature cycles are unlikely to be due to rotational motions of dyes.

To address the dynamical origins of the observed FRET states on Pro6 and dsDNA molecules, we compare their timescales in the following. In Pro6, the dark state appeared frequently and occupied almost half the time of the trace. Moreover, the transitions from the dark-state to both high- 
FRET and low-FRET states were also observed frequently. Dozens of transitions between the three states took place in 700 temperature-cycles of $20 \mu \mathrm{s}$. Furthermore, each state occupied at least several tens (10-100) of snapshots before a transition to a different state occurred. This clearly indicates that the conformation changes inducing the transitions are relatively slow. By the same scaling argument as above, we speculate that this dynamics would correspond to sub-microsecond timescales in water solution. On the contrary, dsDNA molecules were in the high-FRET state most of the time and showed only occasional transitions to the low-FRET state for short times. During 3200 temperature-cycles of $20 \mu \mathrm{s}$, ten transitions between high-FRET and low-FRET states took place. We also noted that the low-FRET state in dSDNA was rare and shortlived (less than 50 temperature-cycles of $20 \mu \mathrm{s}$ ). These observations indicate that such transitions between different FRET states happen on a similar timescale in dsDNA, but with a much lower probability.

The difference in timescales of the dark state and low-FRET state between Pro6 and dsDNA is most likely due to the interactions between linkers and biomolecules, as suggested by molecular dynamics simulations in water. ${ }^{28,31}$ Even though the quantitative extent of such interactions is difficult to predict in view of the limitations of the accuracy of current force fields and the large concentration of glycerol in our experiments, the long and flexible linker can bend in and come into contact with polyproline and this process is much slower than the rotational motions of dyes. ${ }^{28,31}$ However, conformations with folded linkers can bring the donor and the acceptor at short distances and influence their interactions.

Moreover, evidence on dye-dye quenching at short dye-dye separations is also found in polyproline and dsDNA samples at room temperature in aqueous solutions. We observed an additional decay component in nanosecond fluorescence correlation spectroscopy (FCS) measurements on Pro6 in aqueous solution at room temperature (Fig. S4, ESI $\dagger$ ). This extra correlation decays on a time scale of $\sim 100 \mathrm{~ns}$, which is likely due to static dye-dye quenching ${ }^{62}$ at short separations since it is absent in Pro20 samples, where the polyproline helix is too long for the dyes to get into contact. Fitting the FCS data with a model function that takes into account three dynamic components including antibunching, dye-dye quenching and triplet blinking, ${ }^{63-66}$ we found a quenching component of 85 ns. Taking the viscosity difference between glycerol and water into account, the time scale of this correlation agrees with that we found in our temperature-cycle measurements on Pro6 (several tens of snapshots). In addition, specific bunchings in fluorescence autocorrelations at short donor acceptor separations were also noticed in dsDNA samples, as reported by Widengren et al. ${ }^{67}$ Hence, the dye-dye interaction due to overlapping of accessible volumes at short dye-dye separations is a logical consequence.

We therefore attribute the dark state and the low-FRET state to such close encounters of the donor and the acceptor. When the two dyes are separated by distances on the order of their van der Waals radii, their photophysical properties may alter reversibly with new non-radiative relaxation pathways offered by electronic interactions. ${ }^{58}$ Possible charge transfer at short distances may lead to dark states for one or both of the dyes. Whenever this happens to the donor, both the donor and the acceptor will become dark in fluorescence, thus a dark period is observed. If this happens to the acceptor, however, we could still observe fluorescence from the donor, leading to a lowFRET state. The strength of such interactions depends on the separation between the donor and the acceptor. In Pro6 constructs, the donor and the acceptor might be more likely to get close to each other if the donor can stick onto the polyproline chain. ${ }^{28,31}$ Moreover, the dyes are freer to move than in the dsDNA construct. On the contrary, the dyes in dsDNA constructs are less likely to interact with each other because the bulky DNA structure stands in the way. This may explain why the dark state and the low-FRET state appear more frequently and last for longer times in Pro6 constructs than in dsDNA constructs. The multiple FRET states and/or intensity levels may result from the dye-chain interactions, and dye-dye interactions at short interdye separations. The temperature cycles can reveal these intermediate states, and provide deeper insight into the dynamics of these constructs than steady-state singlemolecule measurements alone.

\section{Conclusion}

We demonstrate both steady-state and temperature-cycle experiments on Pro6 and dsDNA samples. By applying temperature cycles, we reveal that complex dynamical processes occurred in these molecules, which were used as models to demonstrate FRET as a "spectroscopic ruler". The multiple FRET states and dynamical transitions between these states show different behaviors in Pro6 and dsDNA samples. Comparing the timescales and probabilities of all these different FRET states, we assign them to conformational heterogeneity that is related to linker dynamics and dye-dye separations. We attribute the multiple FRET states and multi-level intensities to dye-chain and dye-dye interactions at short interdye distances. We have demonstrated the temperature-cycle method as a powerful tool to study heterogeneous dynamics on a singlemolecule scale using FRET. We found dsDNA molecules to better reveal FRET phenomena due to much reduced interaction between the dye-linker and the DNA chain. It would be interesting to further study two-level dynamical systems such as DNA hairpins ${ }^{68-70}$ and Holliday-junctions ${ }^{6,10}$ using the temperature-cycle method in future work.

\section{Acknowledgements}

H.Y., A.G., J.S. and M.O. acknowledge financial support from ERC advanced grant SiMoSoMa. H.Y. acknowledges a postdoctoral fellowship from FWO (Research Foundation Flanders). B.S. was supported by the Swiss National Science Foundation. C.A.M.S. thanks Suren Felekyan for his help in the analysis and Thomas Peulen for generating the accessible volume plot. 


\section{References}

1 L. Stryer and R. P. Haugland, Proc. Natl. Acad. Sci. U. S. A., 1967, 58, 719-726.

2 J. R. Lakowicz, Principles of Fluorescence Spectroscopy, Springer, 3rd edn, 2006.

3 B. Schuler, E. A. Lipman, P. J. Steinbach, M. Kumke and W. A. Eaton, Proc. Natl. Acad. Sci. U. S. A., 2005, 102, 2754-2759.

4 Y. Gambin, V. VanDelinder, A. C. Ferreon, E. A. Lemke, A. Groisman and A. A. Deniz, Nat. Methods, 2011, 8, 239-241.

5 S. A. McKinney, C. Joo and T. Ha, Biophys. J., 2006, 91, 1941-1951.

6 S. Hohng, R. Zhou, M. K. Nahas, J. Yu, K. Schulten, D. M. Lilley and T. Ha, Science, 2007, 318, 279-283.

7 T. Ha, T. Enderle, D. F. Ogletree, D. S. Chemla, P. R. Selvin and S. Weiss, Proc. Natl. Acad. Sci. U. S. A., 1996, 93, 6264-6268.

8 A. A. Deniz, M. Dahan, J. R. Grunwell, T. Ha, A. E. Faulhaber, D. S. Chemla, S. Weiss and P. G. Schultz, Proc. Natl. Acad. Sci. U. S. A., 1999, 96, 3670-3675.

9 B. Schuler, E. A. Lipman and W. A. Eaton, Nature, 2002, 419, 743-747.

10 S. A. McKinney, A. C. Declais, D. M. Lilley and T. Ha, Nat. Struct. Biol., 2003, 10, 93-97.

11 F. Hillger, D. Hanni, D. Nettels, S. Geister, M. Grandin, M. Textor and B. Schuler, Angew. Chem., Int. Ed., 2008, 47, 6184-6188.

12 D. Nettels, S. Muller-Spath, F. Kuster, H. Hofmann, D. Haenni, S. Ruegger, L. Reymond, A. Hoffmann, J. Kubelka, B. Heinz, K. Gast, R. B. Best and B. Schuler, Proc. Natl. Acad. Sci. U. S. A., 2009, 106, 20740-20745.

13 W. J. Koopmans, R. Buning, T. Schmidt and J. van Noort, Biophys. J., 2009, 97, 195-204.

14 H. S. Chung, J. M. Louis and W. A. Eaton, Proc. Natl. Acad. Sci. U. S. A., 2009, 106, 11837-11844.

15 A. Gansen, A. Valeri, F. Hauger, S. Felekyan, S. Kalinin, K. Toth, J. Langowski and C. A. M. Seidel, Proc. Natl. Acad. Sci. U. S. A., 2009, 106, 15308-15313.

16 E. Sisamakis, A. Valeri, S. Kalinin, P. J. Rothwell and C. A. M. Seidel, in Methods in Enzymology, Vol 475: Single Molecule Tools, Pt B: Super-Resolution, Particle Tracking, Multiparameter, and Force Based Methods, ed. N. G. Walter, Elsevier Academic Press Inc, San Diego, 2010, vol. 474, pp. 455-514.

17 L. P. Watkins and H. Yang, Biophys. J., 2004, 86, 4015-4029.

18 J. R. Lakowicz, I. Gryczynski, G. Piszczek, L. Tolosa, R. Nair, M. L. Johnson and K. Nowaczyk, Methods Enzymol., 2000, 323, 473-509.

19 K. A. Dill, S. B. Ozkan, T. R. Weikl, J. D. Chodera and V. A. Voelz, Curr. Opin. Struct. Biol., 2007, 17, 342-346.

20 H. S. Chung, K. McHale, J. M. Louis and W. A. Eaton, Science, 2012, 335, 981-984.

21 L. A. Campos, J. Liu, X. Wang, R. Ramanathan, D. S. English and V. Munoz, Nat. Methods, 2011, 8, 143-146.

22 R. Zondervan, F. Kulzer, H. van der Meer, J. Disselhorst and M. Orrit, Biophys. J., 2006, 90, 2958-2969.
23 J. Kubelka, Photochem. Photobiol. Sci., 2009, 8, 499-512.

24 E. D. Holmstrom, N. F. Dupuis and D. J. Nesbitt, Biophys. J., 2014, 106, 220-231.

25 M. E. Polinkovsky, Y. Gambin, P. R. Banerjee, M. J. Erickstad, A. Groisman and A. A. Deniz, Nat. Commun., 2014, 5, 5737.

26 H. Yuan, T. Xia, B. Schuler and M. Orrit, Phys. Chem. Chem. Phys., 2011, 13, 1762-1769.

27 L. P. Watkins, H. Chang and H. Yang, J. Phys. Chem. A, 2006, 110, 5191-5203.

28 R. B. Best, K. A. Merchant, I. V. Gopich, B. Schuler, A. Bax and W. A. Eaton, Proc. Natl. Acad. Sci. U. S. A., 2007, 104, 18964-18969.

29 S. Doose, H. Neuweiler, H. Barsch and M. Sauer, Proc. Natl. Acad. Sci. U. S. A., 2007, 104, 17400-17405.

30 S. Sindbert, S. Kalinin, H. Nguyen, A. Kienzler, L. Clima, W. Bannwarth, B. Appel, S. Muller and C. A. M. Seidel, J. Am. Chem. Soc., 2011, 133, 2463-2480.

31 M. Hoefling, N. Lima, D. Haenni, C. A. M. Seidel, B. Schuler and H. Grubmuller, PLoS One, 2011, 6, 19.

32 D. Badali and C. C. Gradinaru, J. Chem. Phys., 2011, 134, 225102.

33 A. Iqbal, S. Arslan, B. Okumus, T. J. Wilson, G. Giraud, D. G. Norman, T. Ha and D. M. Lilley, Proc. Natl. Acad. Sci. U. S. A., 2008, 105, 11176-11181.

34 J. Ouellet, S. Schorr, A. Iqbal, T. J. Wilson and D. M. Lilley, Biophys. J., 2011, 101, 1148-1154.

35 W. Kugel, A. Muschielok and J. Michaelis, ChemPhysChem, 2012, 13, 1013-1022.

36 H. S. Chung, J. M. Louis and W. A. Eaton, Biophys. J., 2010, 98, 696-706.

37 N. Di Fiori and A. Meller, Biophys. J., 2010, 98, 2265-2272.

38 S. Kalinin, E. Sisamakis, S. W. Magennis, S. Felekyan and C. A. M. Seidel, J. Phys. Chem. B, 2010, 114, 6197-6206.

39 M. Antonik, S. Felekyan, A. Gaiduk and C. A. M. Seidel, J. Phys. Chem. B, 2006, 110, 6970-6978.

40 E. Nir, X. Michalet, K. M. Hamadani, T. A. Laurence, D. Neuhauser, Y. Kovchegov and S. Weiss, J. Phys. Chem. $B, 2006,110,22103-22124$.

41 S. S. Vogel, T. A. Nguyen, B. W. van der Meer and P. S. Blank, PLoS One, 2012, 7, e49593.

42 S. M. Mansfield and G. S. Kino, Appl. Phys. Lett., 1990, 57, 2615-2616.

43 K. Koyama, M. Yoshita, M. Baba, T. Suemoto and H. Akiyama, Appl. Phys. Lett., 1999, 75, 1667-1669.

44 T. D. Milster, J. S. Jo and K. Hirota, Appl. Opt., 1999, 38, 5046-5057.

45 K. Karrai, X. Lorenz and L. Novotny, Appl. Phys. Lett., 2000, 77, 3459-3461.

46 S. Moehl, H. Zhao, B. D. Don, S. Wachter and H. Kalt, J. Appl. Phys., 2003, 93, 6265-6272.

47 T. Schroder, F. Gadeke, M. J. Banholzer and O. Benson, New J. Phys., 2011, 13, 9.

48 M. Celebrano, R. Lettow, P. Kukura, M. Agio, A. Renn, S. Gotzinger and V. Sandoghdar, Opt. Express, 2010, 18, 13829-13835. 
49 Y. L. Rezus, S. G. Walt, R. Lettow, A. Renn, G. Zumofen, S. Gotzinger and V. Sandoghdar, Phys. Rev. Lett., 2012, 108, 093601.

50 S. Fujiyoshi, M. Fujiwara, C. Kim, M. Matsushita, A. M. van Oijen and J. Schmidt, Appl. Phys. Lett., 2007, 91, 3.

51 S. Fujiyoshi, M. Fujiwara and M. Matsushita, Phys. Rev. Lett., 2008, 100, 4.

52 http://www.fluorophores.tugraz.at [Online].

53 M. Maus, M. Cotlet, J. Hofkens, T. Gensch, F. C. De Schryver, J. Schaffer and C. A. M. Seidel, Anal. Chem., 2001, 73, 2078-2086.

54 H. Yuan, S. Khatua, P. Zijlstra and M. Orrit, Faraday Discuss., 2013, 167, 515-527.

55 J. A. Hanson, J. Brokaw, C. C. Hayden, J.-W. Chu and H. Yang, Chem. Phys., 2012, 396, 61-71.

56 D. S. Clark, J. J. Dechter and L. Mandelkern, Macromolecules, 1979, 12, 626-633.

57 C. Grathwohl and K. Wüthrich, Biopolymers, 1981, 20, 2623-2633.

58 R. Brune, S. Doose and M. Sauer, Biophotonics 2007 Conference, Munich, Germany, 2007.

59 K. Schroter and E. Donth, J. Chem. Phys., 2000, 113, 9101-9108.

60 R. E. Dale, J. Eisinger and W. E. Blumberg, Biophys. J., 1979, 26, 161-193.
61 J. Eisinger, B. Feuer and A. A. Lamola, Biochemistry, 1969, 8, 3908-3915.

62 K. Chattopadhyay, E. L. Elson and C. Frieden, Proc. Natl. Acad. Sci. U. S. A., 2005, 102, 2385-2389.

63 A. Soranno, B. Buchli, D. Nettels, R. R. Cheng, S. MüllerSpäth, S. H. Pfeil, A. Hoffmann, E. A. Lipman, D. E. Makarov and B. Schuler, Proc. Natl. Acad. Sci. U. S. A., 2012, 109, 17800-17806.

64 D. Nettels, I. V. Gopich, A. Hoffmann and B. Schuler, Proc. Natl. Acad. Sci. U. S. A., 2007, 104, 2655-2660.

65 D. Nettels, A. Hoffmann and B. Schuler, J. Phys. Chem. B, 2008, 112, 6137-6146.

66 C. Zander, J. Enderlein and R. A. Keller, in Single Molecule Detection in Solution: Methods and Applications, ed. C. Zander, J. Enderlein and R. A. Keller, Wiley-VCH Verlag, 2003.

67 J. Widengren, E. Schweinberger, S. Berger and C. A. M. Seidel, J. Phys. Chem. A, 2001, 105, 6851-6866.

68 J. P. Torella, S. J. Holden, Y. Santoso, J. Hohlbein and A. N. Kapanidis, Biophys. J., 2011, 100, 1568-1577.

69 T. E. Tomov, R. Tsukanov, R. Masoud, M. Liber, N. Plavner and E. Nir, Biophys. J., 2012, 102, 1163-1173.

70 M. I. Wallace, L. M. Ying, S. Balasubramanian and D. Klenerman, J. Phys. Chem. B, 2000, 104, 11551-11555. 